\title{
ABOUT THE ORIGINS OF THE SUPERSYMMETRIC STANDARD MODEL
}

\author{
P. Fayet ${ }^{\mathrm{a} *}$ \\ a Laboratoire de Physique Théorique de l'Ecole Normale Supérieure \\ 24 rue Lhomond, 75231 Paris Cedex 05, France
}

Invited talk at the International Symposium "30 Years of Supersymmetry"

Minneapolis, Oct. 13-15, 2000

Could one use supersymmetry to relate the fermions, constituants of matter, with the bosons messengers of the interactions? This is, ideally, what a symmetry between fermions and bosons would be expected to do.

However many obstacles seemed, long ago, to prevent supersymmetry from possibly being a fundamental symmetry of Nature. Which fermions and bosons could be related? Is spontaneous supersymmetry breaking possible at all? If yes, where is the corresponding spin- $\frac{1}{2}$ Goldstone fermion? Supersymmetric theories also involve Majorana fermions, unknown in Nature. And how could we define conserved quantum numbers like $B$ and $L$, when these are carried by fundamental (Dirac) fermions only, not by bosons?

An early attempt to relate the photon with a "neutrino" led us to $R$-invariance and to a new $R$ quantum number carried by the supersymmetry generator, but this "neutrino" had to be reinterpreted as a new particle, the photino. We also had to introduce bosons carrying "fermion numbers" $B$ and $L$, which became the squarks and sleptons. This led to the Supersymmetric Standard Model, involving $S U(3) \times S U(2) \times U(1)$ gauge superfields interacting with chiral quark and lepton superfields, and two doublet Higgs superfields responsible for quark and lepton masses. $R$-parity, deeply related with $B$ and $L$ conservation laws, appeared as a remnant of the original $R$-invariance, reduced to a discrete symmetry so that the gravitino and gluinos can acquire masses. We also comment about supersymmetry breaking.

\section{GENERAL OVERVIEW}

Where is the idea of a "Superworld" coming from? Could half of the particles, at least, have escaped our direct observations?

According to common wisdom supersymmetry is an algebraic structure which allows in principle to relate particles of half-integer spins,

*UMR 8549, Unité Mixte du CNRS et de l'Ecole Normale Supérieure.

LPTENS/01-34 namely fermions, with particles of integer spins, which are bosons. Could one relate the fermions, constituants of matter, with the bosons, which appear as the messengers of interactions, and then arrive at some sort of unification between Matter and Forces? This would certainly be very attractive, but unfortunately things are not so simple.

Let's go back in time, about thirty years ago. The algebraic structure of supersymmetry in four dimensions, whose origins in the East and in the West were related in details at this Conference, 
was introduced in the beginning of the seventies by Gol'fand and Likhtman [1], Volkov and Akulov [2], and Wess and Zumino [3]. It involves a spin- $\frac{1}{2}$ fermionic symmetry generator relating fermionic with bosonic fields, in relativistic quantum field theories.

Supersymmetry essentially appeared, at the beginning, as a rather formal mathematical structure. At that time it was not at all clear if, and even less how, supersymmetry could have a chance to actually relate fermions with bosons, in a physical theory of particles. Even more, although supersymmetry is commonly known as relating fermions with bosons, its algebra does not even require the existence of elementary bosons at all! (In the framework of non-linear supersymmetry a fermionic field can be turned into a composite bosonic field made of two fermionic ones [2].) In any case, supersymmetry as an algebraic structure does not by itself require the existence of superpartners for all particles, to which we are so accustomed now.

Considering an algebra is not sufficient to give it a physical meaning. The existence of $S U(2)$ as a mathematical structure does not mean isospin or weak-interaction symmetries, charm is not a consequence of $S U(4)$, nor grand-unification a prediction of $S U(5) \ldots$ An algebra may be of interest, in physics, depending on if and how it can actually be used to describe the real world. In the early days of supersymmetry the hopes were more like using it as a tool to understand better the properties of relativistic field theories; or trying to relate amplitudes involving integerspin mesons with those involving half-integer-spin baryons; or at best, if supersymmetry could actually be used in the description of the real world and were to be realized at a fundamental level, to attempt to use it to relate spin-1 gluons with spin- $\frac{1}{2}$ quarks, for example. However, while very interesting from the point of view of relativistic field theory, supersymmetry seemed, in the early days, clearly inappropriate for a description of our physical world, for obvious and less obvious reasons, which often tend to be somewhat forgotten, now that we got so accustomed to deal with $\mathrm{Su}-$ persymmetric extensions of the Standard Model.
At first, one could not identify physical bosons and fermions that might be related under such a symmetry, in contrast with other symmetries which relate known particles together, such as isospin, electroweak-interaction or grandunification symmetries. Even if it still remains hypothetical, the grand-unification symmetry, for example, should relate known leptons with known quarks, while supersymmetry does not seem to allow for similar relations between known bosons and known fermions.

It even seemed initially that supersymmetry could not be spontaneously broken at all - also in contrast with ordinary symmetries like internal or gauge symmetries - which would imply that bosons and fermions be systematically degenerated in mass! Unless of course supersymmetrybreaking terms are explicitly introduced "by hand", which would spoil the fundamental rôle of the supersymmetry and prevent it from being realized as a local gauge symmetry. In any case, we know significantly more fundamental fermion fields, describing leptons and quarks, than boson fields. To help better set the stage, let us recall that at the time, in 1974, only two fermion families were known and not even complete with the charm quark still to be discovered, neutral current effects had just been discovered the year before, in 1973 , with very little information available about the structure of the weak neutral current(s?), and the lower limit on the mass of a charged $W$ boson was something like $5 \mathrm{GeV} / c^{2}$. The Standard Model was a recent theoretical construction, far from "Standard" in today's sense; its $W^{ \pm}$and $Z$ bosons, of course hypothetical, were considered as really very heavy; and even-more-hypothetical Higgs fields were generally viewed as a technical device to trigger or mimic the spontaneous breaking of the gauge symmetry.

Furthermore, independently of the previous problems of supersymmetry, these theories also involve, systematically, self-conjugate Majorana spinors, while Majorana fermions are completely unknown in Nature - the fermions that we know all appearing as Dirac fermions carrying conserved quantum numbers, $B$ and $L$. Speaking of $B$ and $L$, how could we account for the conservation of these "fermionic numbers" 
(only carried by fermions) in a supersymmetric theory, in which fermions are related to bosons? Should some bosons carry fermionic number and what about "fermion number" conservation, especially if such bosons carrying "fermion number" could be exchanged between quarks and leptons? In view of all these problems, or even without being explicitly aware of them, most physicists considered supersymmetry as irrelevant for "real physics".

Still this algebraic structure could be taken seriously as a possible symmetry of the physics of fundamental particles and interactions, once we understood that the above obstacles preventing the application of supersymmetry to the real world could be overcome. In particular the last questions about the conservation of quantum numbers led us to consider the possibilities of having an additive quantum number carried by the supersymmetry generator - and this was the $R$ quantum number associated with the continuous " $R$-invariance", also implying restrictions on the allowed superpotential - and to have bosons carrying "fermion numbers" $B$ and $L$, which became the squarks and sleptons.

After an initial attempt illustrating how far one could go in trying to relate known particles together (in particular the photon with a "neutrino", and the $W^{ \pm}$'s with charged "leptons", also related with charged Higgs bosons $H^{ \pm}$), and the limitations of this approach, in a $S U(2) \times$ $U(1)$ electroweak theory involving two doublet Higgs superfields now known as $H_{1}$ and $H_{2}$ [ [ ], we were quickly led to reinterpret the fermions of this model (which all possess \pm 1 unit of a conserved additive $R$ quantum number carried by the supersymmetry generator) as belonging to a new class of particles. The "neutrino" ought to be considered as a really new particle, a "photonic neutrino", a name I transformed in 1977 into photino; the fermionic partners of the colored gluons (quite distinct from the quarks) then becoming the gluinos, and so on. More generally this led us to postulate the existence of new $R$-odd "superpartners" for all particles and consider them seriously, despite their rather nonconventional properties: e.g. new bosons carrying "fermion" number, now known as sleptons and squarks, or Majorana fermions transforming as an $S U(3)$ color octet, which are precisely the gluinos, etc.. In addition the electroweak breaking must be induced by a pair of electroweak Higgs doublets, not just a single one as in the Standard Model, which requires the existence of charged Higgs bosons, and of several neutral ones [5, 6].

This construction illustrates that supersymmetry is the framework in which fundamental spin-0 (Englert-Brout) Higgs fields find their natural place. In ordinary gauge theories spin-0 Higgs fields were generally considered as ad hoc additions to the sectors of spin-1 gauge bosons and spin- $\frac{1}{2}$ fermions, and much effort was subsequently devoted at attempting to avoid them, as in technicolor theories for example (which still could not get rid of them completely, independently of other problems). In this framework of supersymmetry fundamental spin-0 fields are taken seriously from the beginning, exactly on the same footing as spin- $\frac{1}{2}$ fields which appear as their fermionic counterparts under supersymmetry. The number of categories of fundamental objects is decreased from 3 in ordinary gauge theories (spin-1 gauge bosons interacting with spin- $\frac{1}{2}$ fermions and spin-0 Higgs fields) to 2 only in supersymmetric gauge theories: (spin-1/ spin- $\left.\frac{1}{2}\right)$ multiplets of "gauge particles" interacting with (spin- $\frac{1}{2} /$ spin-0) "chiral" multiplets describing spin-0 Higgs fields as well as spin- $\frac{1}{2}$ lepton and quark fields - although they are not directly related by the supersymmetry. (Indeed a new discrete symmetry known as $R$-parity comes in to distinguish, among spin- $\frac{1}{2} /$ spin- 0 chiral multiplets, the two separate sectors of Higgs multiplets on one hand, and quark and lepton multiplets, on the other hand.)

In the same manner the number of categories of coupling constants gets also reduced down to 2 , namely gauge and Yukawa couplings only. The Higgs potential in a supersymmetric theory is now determined by the gauge and Yukawa couplings of spin- $\frac{1}{2}$ particles, with Higgs mass parameters identical to fermion mass parameters up to supersymmetry-breaking contributions (naturally expected to be $\lesssim$ electroweak scale given their interplay with Higgs v.e.v.'s, if no excessive 
fine-tuning is to be performed). In particular, the quartic couplings of the two Higgs doublets described by the superfields $H_{1}$ and $H_{2}$ mentioned earlier, as they appear for example in the "minimal" version of the Supersymmetric Standard Model, are completely fixed by the supersymmetry in terms of $g^{2}$ and $g^{2}+g^{\prime 2}\left(g\right.$ and $g^{\prime}$ being the $S U(2) \times U(1)$ electroweak gauge couplings), a fact at the origin of various mass relations involving massive spin-1 gauge bosons and spin-0 Higgs bosons.

The still-hypothetical superpartners may be distinguished by a new quantum number called $R$-parity, first defined in terms of the previous $R$ quantum number as $R_{p}=(-1)^{R}$, i.e. +1 for the ordinary particles and -1 for their superpartners. It is associated with a $Z_{2}$ remnant of the previous $R$-symmetry acting continuously on gauge, lepton, quark and Higgs superfields as in [ [], which must be be abandoned as a continuous symmetry so as to allow for the gravitino [ 6] and gluinos [ [] - upon which the continuous $R$-symmetry acts chirally - to acquire masses (whatever is the actual mechanism, still unknown, ultimately responsible for the spontaneous breaking of the supersymmetry). This new discrete quantum number defined as $R$ parity [ 7] may be multiplicatively conserved in a natural way, and is especially useful to guarantee the absence of unwanted interactions mediated by squark or slepton exchanges - in connection with the fact that $R$-symmetries imply restrictions on the allowed superpotential, actually permitting, in the present case, all useful superpotential terms necessary to generate quark and lepton masses, while excluding dangerous $B$ and $L$-violating terms. The conservation (or nonconservation) of $R$-parity is therefore closely related with the conservation (or non-conservation) of baryon and lepton numbers, $B$ and $L$, as illustrated by the well-known formula reexpressing $R$-parity in terms of baryon and lepton numbers, as $(-1)^{2 S}(-1)^{3 B+L}[$ [8] .

The finding of the basic building blocks of the Supersymmetric Standard Model, whether "minimal" or not, allowed for the experimental searches for "supersymmetric particles", which started with the first searches for gluinos and photinos, selectrons and smuons, in the years 1978-1980, and have been going on continuously since. These searches often use the "missing energy" signature corresponding to energy-momentum carried away by unobserved neutralinos [ 5, 8, 9, 10]. A conserved $R$-parity also ensures the stability of the "lightest supersymmetric particle", a good candidate to constitute the non-baryonic Dark Matter that seems to be present in the Universe.

The general opinion of the scientific community towards supersymmetry and supersymmetric extensions of the Standard Model has considerably changed since the early days, in view of all the nice features of such theories, including their fundamental relation with gravity, their improved renormalisation properties (with the softening or cancellation of divergencies between boson and fermion contributions), the effects of the new particles on the high-energy evolution of gauge couplings, the rôle of supersymmetry in the consistency of (super)string theories, etc.. And it is now widely admitted that supersymmetry may well be the next fundamental symmetry to be discovered in the physics of fundamental particles and interactions, although this remains to be experimentally proven.

\section{NATURE DOES NOT SEEM TO BE SUPERSYMMETRIC !}

The supersymmetry algebra

$$
\left\{\begin{array}{c}
\{Q, \bar{Q}\}=-2 \gamma_{\mu} P^{\mu}, \\
{\left[Q, P^{\mu}\right]=c}
\end{array}\right.
$$

was introduced, in the years 1971-1973, by three different groups, with quite different motivations. Gol'fand and Likhtman [ [1] first introduced it with the hope of understanding parityviolation: when the Majorana supersymmetry generator is written as a two-component chiral Dirac spinor (say $Q_{L}$ ), one may have the impression that the supersymmetry algebra, which then involves a chiral projector in the right-handside of the anticommutation relation (11), is intrinsically parity-violating; they suggested that such models must therefore necessarily violate parity, probably thinking this could lead to an explanation for parity-violation in weak interactions. Volkov 
and Akulov [ 2] hoped to explain the masslessness of the neutrino from a possible interpretation as a spin- $\frac{1}{2}$ Goldstone particle, while Wess and Zumino [3] wrote the algebra by extending to four dimensions the "supergauge" (i.e. supersymmetry) transformations [ 11], and algebra [12], acting on the two-dimensional string worldsheet. However, the mathematical existence of an algebraic structure does not imply that it has to play a rôle as an invariance of the fundamental laws of Nature.

Indeed many obstacles seemed, long ago, to prevent supersymmetry from possibly being a fundamental symmetry of Nature. Which bosons and fermions could be related by supersymmetry? May be supersymmetry could act at the level of composite objects, e.g. as relating baryons with mesons? Or should it act at a fundamental level, i.e. at the level of quarks and gluons? But quarks are color triplets, and electrically charged, while gluons transform as an $S U(3)$ color octet, and are electrically neutral! Is spontaneous supersymmetry breaking possible at all? If yes, where is the spin- $\frac{1}{2}$ Goldstone fermion of supersymmetry, if not one of the known neutrinos? Can we use supersymmetry to relate directly known bosons and fermions? And, if not, why? If known bosons and fermions cannot be directly related by supersymmetry, do we have to accept this as the sign that supersymmetry is not a symmetry of the fundamental laws of Nature? If we still insist to work within the framework of supersymmetry, how could it be possible to define conserved baryon and lepton numbers in such theories, which systematically involve self-conjugate Majorana fermions, unknown in Nature, while $B$ and $L$ are carried only by fundamental (Dirac) fermions - not by bosons ? And, once we are finally led to postulate the existence of new bosons carrying $B$ and $L$ - the new spin-0 squarks and sleptons - can we prevent them from mediating new unwanted interactions, which would have disastrous effects?

While bosons and fermions should have equal masses in a supersymmetric theory, this is certainly not the case in Nature. Supersymmetry should then clearly be broken. But spontaneous supersymmetry breaking is notoriously difficult to achieve, to the point that it was even initially thought to be impossible! Why is it so? Here it is important to point out an important difference between supersymmetry and other symmetries such as internal or gauge symmetries for example. To break spontaneously such "ordinary" symmetries, it is sufficient to arrange so that the symmetry-preserving would-be vacuum state has more energy than symmetry-breaking vacua, so that it gets unstable. This is for example how the electroweak symmetry gets spontaneously broken in the Standard Model, as soon as the Higgs mass parameter $\mu^{2}$ is taken to be negative.

But in supersymmetry one no longer has the same freedom to fix at will the potential of scalar fields as in ordinary gauge theories, since this potential is now largely determined by gauge and Yukawa couplings. Furthermore supersymmetry is in fact a very special symmetry, since the Hamiltonian, which governs the determination of the vacuum state through the minimization of the potential, is also directly related by the supersymmetry algebra itself to the supersymmetry generator, precisely the one that we would like to see spontaneously broken. Actually this hamiltonian $H$, which appears in the right-handside of the anticommutation relations (11), can be expressed proportionally to the sum of the squares of the components of the supersymmetry generator, as $H=\frac{1}{4} \sum_{\alpha} Q_{\alpha}^{2}$. This implies that a supersymmetry preserving vacuum state must have vanishing energy, while any candidate for a "vacuum state" which would not be invariant under supersymmetry may naïvely be expected to have a larger, positive, energy [ 13] 2. As a result, potential candidates for supersymmetry breaking vacuum states seemed to be necessarily unstable, leading to the question:

\section{Is spontaneous supersymmetry breaking possible at all?}

In spite of the above argument, several ways of breaking spontaneously global or local supersymmetry have been found. But spontaneous super-

\footnotetext{
${ }^{2}$ Such a would-be supersymmetry breaking state corresponds, in global supersymmetry, to a strictly positive energy density - the scalar potential being expressed proportionally to the sum of the squares of the auxiliary $D, F$ and $G$ components, as $V=\frac{1}{2} \sum\left(D^{2}+F^{2}+G^{2}\right)$.
} 
symmetry breaking remains in general rather difficult to obtain, at least for global supersymmetry, due to the strong tendency of such theories to resist spontaneous supersymmetry breaking by preferring systematically supersymmetric vacua. Only in very exceptional situations can the existence of such vacua be completely avoided! Gauge symmetries, on the other hand, get rather easily (even sometimes too easily) spontaneously broken, in supersymmetric theories.

In global supersymmetry a non-supersymmetric state has, in principle, always more energy than a supersymmetric one; it then seems that it should always be unstable! Still it is possible to escape this general result - and this is the key to spontaneous supersymmetry breaking - if one can arrange to be in one of those rare situations for which no supersymmetric state exists at all the set of equations for the auxiliary field v.e.v.'s $\langle D\rangle^{\prime} \mathrm{s}=\langle F\rangle^{\prime} \mathrm{s}=\langle G\rangle^{\prime} \mathrm{s}=0$ having no solution at all. But these situations are in general quite exceptional. (This is in sharp contrast with ordinary symmetries, in particular gauge symmetries, for which it is sufficient to arrange for non-symmetric states to have less energy than symmetric ones, which is easy to achieve.) These rare situations usually involve an abelian $U(1)$ gauge group [ 14], allowing for a linear " $\xi D$ " term in the Lagrangian density 3 ; and/or an appropriate set of chiral superfields with special superpotential interactions which must be very carefully chosen [ 15], preferentially with the help of additional symmetries such as $R$-symmetries. In local supersymmetry [16], which includes gravity, one also has to arrange, at the price of a very severe fine-tuning, for the energy density of the vacuum to vanish exactly [17], or almost exactly, to an extremely good accuracy, so as not to generate an unacceptably large value of the cosmological constant $\Lambda$.

Whatever the mechanism of supersymmetry breaking, we still have to get a physical world which looks like ours (which will lead to pos-

\footnotetext{
${ }^{3}$ Even in the presence of such a term, one frequently still does not get a spontaneous breaking of the supersymmetry: one has to be very careful so as to avoid the presence of supersymmetry restoring vacuum states with vanishing energy, which generally tend to exist.
}

tulate the existence of the superpartners). Of course just accepting the possibility of explicit supersymmetry breaking without worrying too much about the origin of supersymmetry breaking terms, as is frequently done now, makes things much easier - but also at the price of introducing a large number of arbitrary parameters, coefficients of these supersymmetry breaking terms. In any case such terms must have their origin in a spontaneous supersymmetry breaking mechanism, if we want supersymmetry to play a fundamental rôle, especially if it is to be realized as a local fermionic gauge symmetry, as in supergravity theories. We shall come back to this question of supersymmetry breaking later. In between, we note that the spontaneous breaking of the global supersymmetry must in any case generate a massless spin- $\frac{1}{2}$ Goldstone particle, leading to the next question,

$$
\begin{gathered}
\text { Where is the spin- } \frac{1}{2} \text { Goldstone fermion } \\
\text { of supersymmetry? }
\end{gathered}
$$

Could it be one of the known neutrinos [2] ? A first attempt at implementing this idea within a $S U(2) \times U(1)$ electroweak model of "leptons" [ - 4 quickly illustrated that it could not be pursued very far. The "leptons" of this first electroweak model were soon reinterpreted to become the "charginos" and "neutralinos" of the Supersymmetric Standard Model.

If the Goldstone fermion of supersymmetry is not one of the known neutrinos, why hasn't it been observed? Today we tend not to think at all about the question, since: 1) the generalized use of soft terms breaking explicitly the supersymmetry seems to make this question irrelevant; 2) since supersymmetry has to be realized locally anyway, within the framework of supergravity [ 16], the massless spin- $\frac{1}{2}$ Goldstone fermion ("goldstino") should in any case be eliminated in favor of extra degrees of freedom for a massive spin- $\frac{3}{2}$ gravitino [ 6, 17.

But where is the gravitino, and why has no one ever seen a fundamental spin- $\frac{3}{2}$ particle? Should this already be taken as an argument against supersymmetry and supergravity theories? Indeed should one consider that the crucial test of such theories should be the discovery of a new funda- 
mental spin- $\frac{3}{2}$ particle? In that case, how could it manifest its presence?

To discuss this question properly we need to know how this spin- $\frac{3}{2}$ particle should couple to the other ones, which requires us to know how bosons and fermions could be associated under supersymmetry [ 5]. In any case, we might already anticipate that the interactions of the gravitino, with amplitudes proportional to the square root of the Newton constant $\sqrt{G_{N}} \simeq$ $10^{-19} \mathrm{GeV}^{-1}$, should be absolutely negligible in particle physics. Quite surprisingly this may, however, not necessarily be true, despite the extreme smallness of Newton's constant! If the spin- $\frac{3}{2}$ gravitino turns out to be light (which is the case if supersymmetry is broken "at the electroweak scale" or even at some larger intermediate scale) it would still interact very much like the massless spin- $\frac{1}{2}$ goldstino of global supersymmetry, according to the "equivalence theorem" of supersymmetry [ 6]. We are then back again to our initial question, where is the spin- $\frac{1}{2}$ Goldstone fermion of supersymmetry? But we are now in a position to answer, the direct detectability of the gravitino depending crucially on the value of its mass $m_{3 / 2}$, itself fixed (according to the relation $m_{3 / 2}=\kappa d / \sqrt{6}$ ) by the supersymmetry breaking scale $\sqrt{d}=\Lambda_{s s} \approx$ $\sqrt{m_{3 / 2} m_{\text {Planck }}}$ [ 6, 18. In particular the gravitino gets essentially "invisible" in particle physics experiments, as soon as the supersymmetry breaking scale is large enough, compared to the electroweak scale. This seems indeed the most likely situation. On the other hand, a sufficiently light gravitino could be directly detectable in particle physics experiments!

In any case, irrespective of the question of supersymmetry breaking, the crucial question, if supersymmetry is to be relevant in particle physics, is:

$$
\begin{aligned}
& \text { Which bosons and fermions } \\
& \text { could be related by supersymmetry? }
\end{aligned}
$$

But there seems to be no answer since known bosons and fermions do not appear to have much in common - excepted, maybe, for the photon and the neutrino. This track deserved to be ex- plored [ [], but one cannot really go very far in this direction. In a more general way the number of (known) degrees of freedom is significantly larger for the fermions (now 90, for three families of quarks and leptons) than for the bosons (27 for the gluons, the photon and the $W^{ \pm}$and $Z$ bosons, ignoring for the moment the spin-2 graviton and the not-yet-discovered Higgs boson). And these fermions and bosons have very different gauge symmetry properties!

As we have already mentioned, supersymmetric theories also involve, systematically, selfconjugate Majorana spinors - unobserved in $\mathrm{Na}-$ ture - while the fermions that we know all appear as Dirac fermions carrying conserved $B$ and $L$ quantum numbers. This leads to the question

$$
\begin{gathered}
\text { How could one define (conserved) } \\
\text { baryon and lepton numbers, } \\
\text { in a supersymmetric theory? }
\end{gathered}
$$

These quantum numbers, presently known to be carried by fundamental fermions only, not by bosons, seem to appear in Nature as intrinsicallyfermionic numbers. Such a feature cannot be maintained in a supersymmetric theory, and one has to accept the (then rather heretic) idea of attributing baryon and lepton numbers to fundamental bosons, as well as to fermions. These new bosons carrying $B$ or $L$ are the superpartners of the spin- $\frac{1}{2}$ quarks and leptons, namely the now-familiar (although still unobserved) spin-0 squarks and sleptons. Altogether, all known particles should be associated with new superpartners [5].

Nowadays we are so used to deal with spin-0 squarks and sleptons, carrying baryon and lepton numbers almost by definition, that we can hardly imagine this could once have appeared as a problem. Its solution went through accepting the idea of attributing baryon or lepton numbers to a large number of new fundamental bosons. But if such new spin- 0 squarks and sleptons are introduced, their direct (Yukawa) exchanges between ordinary quarks and leptons, if allowed, could lead to an immediate disaster, preventing us from getting a theory of electroweak and strong interactions mediated by spin-1 gauge bosons only (and 
not spin-0 particles), with conserved $B$ and $L$ quantum numbers!

$$
\begin{gathered}
\text { How can we avoid unwanted interactions } \\
\text { mediated by spin-0 squark and slepton } \\
\text { exchanges? }
\end{gathered}
$$

Fortunately, we can naturally avoid such unwanted interactions, thanks to $R$-parity (a discrete remnant of the continuous $U(1) R$ symmetry) which, if present, guarantees that squarks and sleptons cannot be directly exchanged between ordinary quarks and leptons, allowing for conserved baryon and lepton numbers in supersymmetric theories.

\section{R-INVARIANCE, AND ELECTRO- WEAK BREAKING}

Let us now return to an early attempt at relating existing bosons and fermions together [ [4], also at the origin of the definition of the continuous $R$-invariance (the discrete version of which

${ }^{4}$ This model is reminiscent of a presupersymmetry model
involving two Higgs doublets and (associated) fermion
doublets, with Yukawa and $\varphi^{4}$ interactions already re-
stricted by a continuous $Q$-invariance in a way which pre-
pares for these Higgs and fermion doublets to get related
by a supersymmetry [ 19 . One unit of $Q$ is then carried
by the supersymmetry generator. These $Q$ transforma-
tions act on gauge and Higgs superfields as follows:

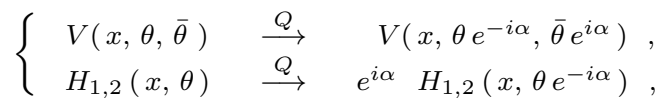

allowing for a direct $\mu H_{1} H_{2}$ Higgs superfield mass term in the superpotential. The definition of $Q$-invariance was then modified, so that it survives the spontaneous breaking of the electroweak symmetry [ [4]. This led to $R$ invariance, acting as follows:

$$
\left\{\begin{array}{ccc}
V(x, \theta, \bar{\theta}) & \stackrel{R}{\longrightarrow} & V\left(x, \theta e^{-i \alpha}, \bar{\theta} e^{i \alpha}\right), \\
H_{1,2}(x, \theta) & \stackrel{R}{\longrightarrow} & H_{1,2}\left(x, \theta e^{-i \alpha}\right) .
\end{array}\right.
$$

The direct $\mu H_{1} H_{2}$ mass term (with $R$-index $n=0$ ), now forbidden by $R$-invariance, was replaced by a trilinear coupling to an extra singlet chiral superfield $N$ transforming as $N(x, \theta) \stackrel{R}{\longrightarrow} e^{2 i \alpha} N\left(x, \theta e^{-i \alpha}\right)$, with an " $R$-invariant" superpotential written, in modern notations, as

$$
\mathcal{W}=\lambda H_{1} H_{2} N+\sigma N .
$$

It has $R$-index $n=2$ and transforms according to eq. (16) of section 5 , so that it generates $R$-invariant interactions. Both the electroweak symmetry and the supersymmetry get spontaneously broken. leading to $R$-parity). It also showed how one can break spontaneously the $S U(2) \times U(1)$ electroweak symmetry in a supersymmetric theory, using a pair of chiral doublet Higgs superfields that would now be called $H_{1}$ and $H_{2}$. This involves a mixing angle (initially called $\delta$ ) known as $\beta$, defined by

$\tan \beta=\frac{v_{2}}{v_{1}}$.

The fermions of this early supersymmetric model, which are in fact gaugino-higgsino mixtures, should no longer be considered as lepton candidates, but became essentially the "charginos" and "neutralinos" of the Supersymmetric Standard Model [5, 6].

Despite the general lack of similarities between known bosons and fermions, we tried as an exercise to see how far one could go in attempting to relate the spin- 1 photon with a spin- $\frac{1}{2}$ neutrino. If we want to attempt to identify the companion of the photon as being a "neutrino", despite the fact that it initially appears as a self-conjugate Majorana fermion, we need to understand how this particle could carry a conserved quantum number that we might interpret as a "lepton" number. This was made possible through to the definition of a continuous $U(1) \quad R$-invariance [ [4], which also guaranteed the masslessness of this "neutrino" ( $\nu_{L}$ ", carrying +1 unit of $R$ ), by acting chirally on it, i.e. also by acting chirally on the Grassmann coordinate $\theta$ which appears in the expression of gauge and chiral superfields. The supersymmetry generator $Q_{\alpha}$ carries one unit of the corresponding additive conserved quantum number, called $R$, so that one has $\Delta R= \pm 1$ between a boson and a fermion related by supersymmetry.

Attempting to relate the photon with one of the neutrinos could only be an exercise of limited validity. The would-be "neutrino", while having in this model a $V-A$ coupling to its associated "lepton" and the charged $W^{ \pm}$boson, was in fact what we would now call a "photino", not directly coupled to the $Z$ ! Still this first attempt, which essentially became a part of the Supersymmetric Standard Model, illustrated how one can break spontaneously a $S U(2) \times U(1)$ gauge symmetry in a supersymmetric theory, through an 
electroweak breaking induced by a pair of chiral doublet Higgs superfields, now known as $H_{1}$ and $H_{2}$ ! (Using a single doublet Higgs superfield would have left us with a massless charged chiral fermion, which is evidently unacceptable.) Our previous charged "leptons" were in fact what we now call two winos, or charginos, obtained from the mixing of charged gaugino and higgsino components, as given by the mass matrix

$\mathcal{M}=\left(\begin{array}{cc}\left(m_{2}=0\right) & \frac{g v_{2}}{\sqrt{2}}=m_{W} \sqrt{2} \sin \beta \\ \frac{g v_{1}}{\sqrt{2}}=m_{W} \sqrt{2} \cos \beta & \mu=0\end{array}\right)$,

in the absence of a direct higgsino mass that would have originated from a $\mu H_{1} H_{2}$ mass term in the superpotential f. The whole construction showed that one could deal elegantly with elementary spin-0 Higgs fields (not a very popular ingredient at the time), in the framework of spontaneously-broken supersymmetric theories. Quartic Higgs couplings are no longer arbitrary, but get fixed by the gauge coupling constants - here the electroweak couplings $g$ and $g^{\prime}-$ through the following " $D$-terms" (i.e. $\frac{\vec{D}^{2}}{2}+\frac{D^{\prime 2}}{2}$ ) in the scalar potential given in [何 6 :

$$
\begin{aligned}
& V_{\text {Higgs }}= \\
& \frac{g^{2}}{8}\left(h_{1}^{\dagger} \vec{\tau} h_{1}+h_{2}^{\dagger} \vec{\tau} h_{2}\right)^{2}+\frac{g^{\prime 2}}{8}\left(h_{1}^{\dagger} h_{1}-h_{2}^{\dagger} h_{2}\right)^{2}+\ldots \\
& =\frac{g^{2}+g^{\prime 2}}{8}\left(h_{1}^{\dagger} h_{1}-h_{2}^{\dagger} h_{2}\right)^{2}+\frac{g^{2}}{2}\left|h_{1}^{\dagger} h_{2}\right|^{2}+\ldots
\end{aligned}
$$

This is precisely the quartic Higgs potential of the "minimal" version of the Supersymmetric Standard Model, the so-called MSSM, with its quartic

${ }^{5}$ The $\mu H_{1} H_{2}$ term initially introduced in [ [], which would have broken explicitly the continuous $U(1) R$ invariance then intended to be associated with "lepton" number conservation, was quickly replaced by a $\lambda \mathrm{H}_{1} \mathrm{H}_{2} \mathrm{~N}$ trilinear coupling involving an extra neutral singlet chiral superfield $N$.

${ }^{6}$ With a different denomination for the two Higgs doublets, such that $\varphi^{\prime \prime} \mapsto h_{1}, \quad\left(\varphi^{\prime}\right)^{c} \mapsto h_{2}, \quad \tan \delta=v^{\prime} / v^{\prime \prime} \mapsto$ $\tan \beta=v_{2} / v_{1}$.
Higgs coupling constants equal to

$\frac{g^{2}+g^{\prime 2}}{8}$ and $\frac{g^{2}}{2}$.

The quartic term $\frac{g^{2}}{2}\left|h_{1}^{\dagger} h_{2}\right|^{2}$ in this scalar potential 7 is responsible for the fact that the vacuum expectation values of the two doublet Higgs fields "align", in order to minimize the energy, so that only neutral components of these doublets acquire non vanishing v.e.v.'s., the $U(1)$ subgroup of electromagnetism remaining unbroken, and the photon massless. This term is also responsible, from $\left\langle h_{1,2}^{0}\right\rangle=v_{1,2} / \sqrt{2}$, for the contribution $\frac{1}{4} g^{2}\left(v_{1}^{2}+v_{2}^{2}\right)\left|H^{-}\right|^{2}=m_{W}^{2}\left|H^{-}\right|^{2}$ to the mass ${ }^{2}$ of the charged Higgs bosons $H^{ \pm}$ (initially called $w^{ \pm}$).

Further contributions to this quartic Higgs potential also appear in the presence of additional superfields, such as the neutral singlet chiral superfield $N$ already introduced in this model, which will play an important rôle in the NMSSM, i.e. in "next-to-minimal" or "non-minimal" versions of the Supersymmetric Standard Model. In any case charged Higgs bosons $H^{ \pm}$are present in this framework, as well as several neutral ones, now called $H, h, A, \ldots$, and one gets in general mass relations such as

$m_{H^{ \pm}}^{2}=m_{W}^{2}+$ susy-breaking terms, $\ldots$.

The exact mass spectrum depends of course on the details of the supersymmetry breaking mechanism considered: use of soft-breaking terms, possibly "derived from supergravity", presence or absence of extra- $U(1)$ gauge fields and/or additional chiral superfields, rôle of radiative corrections, etc..

${ }^{7}$ For this quartic contribution to the Higgs potential, the correspondence between old and new notations is as follows:

$\frac{g^{2}}{2}\left(\varphi^{\prime \dagger} \varphi^{\prime} \varphi^{\prime \prime \dagger} \varphi^{\prime \prime}-\varphi^{\prime \dagger} \varphi^{\prime \prime} \varphi^{\prime \prime \dagger} \varphi^{\prime}\right) \equiv \frac{g^{2}}{2}\left|h_{1}^{\dagger} h_{2}\right|^{2}$.

The vanishing of this quantity implies that the v.e.v.'s of the two Higgs doublets are correctly "aligned", with

$<h_{1}>=\left(\begin{array}{c}v_{1} / \sqrt{2} \\ 0\end{array}\right), \quad<h_{2}>=\left(\begin{array}{c}0 \\ v_{2} / \sqrt{2}\end{array}\right)$. 


\section{THE SUPERSYMMETRIC STAN- DARD MODEL}

These two doublet Higgs superfields are precisely the two doublets, now called $H_{1}$ and $H_{2}$, used in 1977 to generate the masses of charged leptons and down quarks (from $<H_{1}>$ ), and of up quarks (from $<H_{2}>$ ), in supersymmetric extensions of the standard model [5]. At the time having to introduce Higgs fields was generally considered as rather unpleasant. While one Higgs doublet was taken as probably unavoidable to get to the standard model or at least simulate the effects of the spontaneous breaking of the electroweak symmetry, having to consider two Higgs doublets, necessitating charged Higgs bosons as well as several neutral ones, was usually considered as a too heavy price, in addition to the "doubling of the number of particles", once considered as an indication of the irrelevance of supersymmetry. As a matter of fact considerable work was devoted for a time on attempts to avoid fundamental spin-0 Higgs fields, before returning to fundamental Higgses, precisely in this framework of supersymmetry.

In the previous $S U(2) \times U(1)$ model [4], it was impossible to view seriously for very long "gaugino" and "higgsino" fields as possible building blocks for our familiar lepton fields. On the contrary they should describe new particles. This led us to consider that all quarks and leptons ought to be associated with new bosonic partners, the spin-0 squarks and sleptons. Gauginos and higgsinos, mixed together by the spontaneous breaking of the electroweak symmetry, correspond to a new class of fermions, now known as "charginos" (cf. the mass matrix of eq. (8)) and "neutralinos". In particular, the partner of the photon under supersymmetry, which cannot be identified with any of the known neutrinos, should be viewed as a new "photonic neutrino", the photino; the fermionic partner of the gluon octet is an octet of selfconjugate Majorana fermions called gluinos, etc. - although at the time colored fermions belonging to octet representations of the color $S U(3)$ gauge group were generally believed not to exist (to the point that one could think of using the absence of such particles as a general constraint to select admissible grand-unified theories [ 20]).

The two doublet Higgs superfields $H_{1}$ and $H_{2}$ introduced previously can now be used to generate quark and lepton masses 8 [ [ the appropriate mass terms we first write the relevant bilinear products of left-handed lepton $(L)$ and quark $(Q)$ doublet superfields, with the conjugates of the right-handed superfields describing right-handed quarks and leptons, now known to be electroweak singlets. The latter are lefthanded superfields denoted as $\bar{E}, \bar{U}$ and $\bar{D}$, in modern notations. The resulting bilinear terms $\bar{E} L, \bar{D} Q$ and $\bar{U} Q$ are then coupled in a supersymmetric and gauge invariant way to the two doublet Higgs superfields $H_{1}$ and $H_{2}$, which leads to the familiar trilinear superpotential

$\mathcal{W}=h_{e} H_{1} \cdot \bar{E} L+h_{d} H_{1} \cdot \bar{D} Q-h_{u} H_{2} \cdot \bar{U} Q$.

The corresponding superpotential interactions are also invariant under continuous $R$-symmetry transformations, as well as under an "extra- $U(1)$ " symmetry, two important features to which we shall return later. We certainly don't attempt at this point to write further terms in the superpotential that would be odd functions of quark and lepton superfields, since we know in advance that they would lead to $B$ and/or $L$-violations, and it would be somewhat foolish to rush to reestablish the problems of $B$ and $L$ conservation laws that were elegantly solved by attributing baryon and lepton numbers to bosons (squarks and sleptons) as well as to fermions. (Such $B$ or $L$-violating terms, in addition, would not be invariant under $R$ nor under the extra $U(1)$ symmetry 9 , but we

\footnotetext{
${ }^{8}$ The correspondance between earlier notations for doublet Higgs superfields, and modern ones, is as follows:

$$
\begin{gathered}
S=\left(\begin{array}{c}
S^{0} \\
S^{-}
\end{array}\right), T=\left(\begin{array}{c}
T^{0} \\
T^{-}
\end{array}\right) \rightarrow H_{1}=\left(\begin{array}{c}
H_{1}^{0} \\
H_{1}^{-}
\end{array}\right), H_{2}=\left(\begin{array}{c}
H_{2}^{+} \\
H_{2}^{0}
\end{array}\right), \\
\text { (left-h.) (right-h.) }
\end{gathered}
$$
}

so that $S$ gets now replaced by $H_{1}$ and $T^{\dagger}$ by $H_{2}$. Furthermore, we originally denoted, generically, by $S_{i}$, lefthanded, and $T_{j}$, right-handed, the chiral superfields describing the left-handed and right-handed spin- $\frac{1}{2}$ quark and lepton fields, together with their spin-0 partners.

${ }^{9}$ It is interesting to note, in particular, that if the ex- 
Table 1

The basic ingredients of the Supersymmetric Standard Model.

1) $S U(3) \times S U(2) \times U(1)$ gauge superfields;

2) chiral superfields for the three quark and lepton families;

3) two doublet Higgs superfields $H_{1}$ and $H_{2}$ responsible for electroweak breaking, and quark and lepton masses, through

4) the trilinear superpotential (12).

shall return later to the fate of these two additional symmetries.)

The vacuum expectation values of the two Higgs doublets generate charged-lepton and down-quark masses (for the Higgs doublet described by $H_{1}$ ), and up-quark masses (for the one described by $H_{2}$ ). They are given, with an appropriate normalization convention, by $m_{e}=h_{e} v_{1} / \sqrt{2}, \quad m_{d}=h_{d} v_{1} / \sqrt{2}$, and $m_{u}=$ $h_{u} v_{2} / \sqrt{2}$, respectively. All this constitutes the basic structure of the Supersymmetric Standard Model, which involves at least the minimal set of ingredients shown in Table 11. Other ingredients, such as a $\mu H_{1} H_{2}$ direct mass term in the superpotential, or an extra singlet chiral superfield $N$ with a trilinear superpotential coupling $\lambda H_{1} H_{2} N+\ldots$ possibly acting as a replacement for a $\mu H_{1} H_{2}$ direct mass term [ [], and/or extra $U(1)$ factors in the gauge group (which could have been responsible for spontaneous supersymmetry breaking) may or may not be present, depending on the particular version of the Supersymmetric Standard Model considered.

In any case, independently of the details of the supersymmetry breaking mechanism ultimately considered, we obtain the minimal particle con-

tra $U(1)$ of eq. (20) is gauged $B, L$ and $R$ become automatic symmetries which follow from the requirement of local gauge invariance.
Table 2

Minimal particle content of the Supersymmetric Standard Model.

\begin{tabular}{|c|c|c|}
\hline Spin 1 & Spin $1 / 2$ & Spin 0 \\
\hline gluons & \multirow{5}{*}{$\begin{array}{c}\text { gluinos } \tilde{g} \\
\text { photino } \tilde{\gamma} \\
------ \\
\text { winos } \widetilde{W}_{1,2}^{ \pm} \\
\operatorname{zinos} \widetilde{Z}_{1,2} \\
\text { higgsino } \tilde{h}^{0}\end{array}$} & \multirow{5}{*}{$\begin{array}{l}\text { Higgs } \\
\text { bosons }\end{array}$} \\
\hline photon & & \\
\hline$W^{ \pm}$ & & \\
\hline \multirow[t]{3}{*}{$Z$} & & \\
\hline & & \\
\hline & $\begin{array}{cc}\text { leptons } & l \\
\text { quarks } & q\end{array}$ & $\begin{array}{ll}\text { sleptons } & \tilde{l} \\
\text { squarks } & \tilde{q}\end{array}$ \\
\hline
\end{tabular}

tent of the Supersymmetric Standard Model, as given in Table 2. Each spin- $\frac{1}{2}$ quark $q$ or charged lepton $l^{-}$is associated with two spin-0 partners collectively denoted by $\tilde{q}$ or $\tilde{l}^{-}$, while a lefthanded neutrino $\nu_{L}$ is associated with a single spin-0 sneutrino $\tilde{\nu}$. We have ignored for simplicity further mixings between the various "neutralinos" described by neutral gaugino and higgsino fields, denoted in this table by $\tilde{\gamma}, \tilde{Z}_{1,2}$ and $\tilde{h}^{0}$. More precisely, all such models include four neutral Majorana fermions at least, corresponding to mixings of the fermionic partners of the two neutral $S U(2) \times U(1)$ gauge bosons (usually denoted by $\tilde{Z}$ and $\tilde{\gamma}$, or $\tilde{W}_{3}$ and $\tilde{B}$ ) and of the two neutral higgsino components $\left(\tilde{h_{1}^{0}}\right.$ and $\left.\tilde{h_{2}^{0}}\right)$. Non-minimal models also involve additional gauginos and/or higssinos.

\section{FROM $R$-INVARIANCE TO $R$-PA- RITY}

Let us return to the definition of the continuous $R$-symmetry, and discrete $R$-parity, transformations. $\quad R$-parity is associated with a $Z_{2}$ subgroup of the group of continuous $U(1) R$ symmetry transformations, acting on the gauge superfields and the two doublet Higgs superfields $H_{1}$ and $H_{2}$ as in [ 4 (cf. footnote 4 in sec- 
tion 3), with their definition extended to quark and lepton superfields so that quarks and leptons carry $R=0$, and squarks and sleptons, $R= \pm 1$ (more precisely, $R=+1$ for $\tilde{q}_{L}, \tilde{l}_{L}$, and $R=-1$ for $\left.\tilde{q}_{R}, \tilde{l}_{R}\right)$ [ 5]. $R$-parity appears in fact as the remnant of this continuous $R$-invariance when gravitational interactions are introduced [ 6], in the framework of local supersymmetry (supergravity). Either the continuous $R$-invariance, or simply its discrete version of $R$ parity, if imposed, naturally forbid the unwanted direct exchanges of the new squarks and sleptons between ordinary quarks and leptons.

These continuous $U(1) R$-symmetry transformations, which act chirally on the anticommuting Grassmann coordinate $\theta$ appearing in the definition of superspace and superfields, act on the gauge and chiral superfields of the Supersymmetric Standard Model as follows ${ }^{10}$ :

\section{Action of continuous $R$-symmetry:}

$$
\left\{\begin{array}{lll}
V(x, \theta, \bar{\theta}) & \stackrel{R}{\longrightarrow} & V\left(x, \theta e^{-i \alpha}, \bar{\theta} e^{i \alpha}\right), \\
H_{1,2}(x, \theta) & \stackrel{R}{\longrightarrow} & H_{1,2}\left(x, \theta e^{-i \alpha}\right), \\
S(x, \theta) & \stackrel{R}{\longrightarrow} & e^{i \alpha} S\left(x, \theta e^{-i \alpha}\right),
\end{array}\right.
$$

for $\left\{\begin{array}{l}S U(3) \times S U(2) \times U(1) \text { gauge superfields, } \\ \text { left-h. Higgs superfields } H_{1} \text { and } H_{2}, \\ \text { left-h. (anti)quark and lepton superfields } \\ S=\{Q, \bar{U}, \bar{D}, L, \bar{E}\},\end{array}\right.$

$$
\text { respectively . }
$$

These transformations are defined so as not to act on ordinary particles, which have $R=0$, while their superpartners have, therefore, $R= \pm 1$.

$\overline{10}$ If we also introduce as in [ 4] an extra neutral singlet chiral superfield $N$ coupled to the two doublet Higgs superfields $H_{1}$ and $H_{2}$, it gets transformed under $R$ as follows:

$$
N(x, \theta) \stackrel{R}{\longrightarrow} e^{2 i \alpha} N\left(x, \theta e^{-i \alpha}\right),
$$

so that the trilinear superpotential coupling $\lambda H_{1} H_{2} \mathrm{~N}$ is " $R$-invariant", in the sense that it transforms according to eq. (16).
They allow us to distinguish between two separate sectors of $R$-even and $R$-odd particles. $R$-even particles include the gluons, photon, $W^{ \pm}$and $Z$ bosons, the various Higgs bosons, quarks and leptons - and the graviton. $R$-odd particles include their superpartners, i.e. the gluinos and the various neutralinos and charginos, squarks and sleptons - and the gravitino (cf. Table 3). According to this first definition, $R$-parity simply appears as the parity of the above additive quantum number $R$, as given by the expression [ 7 :

$$
\begin{aligned}
& R \text {-parity } R_{p}= \\
& (-1)^{R}=\left\{\begin{array}{rc}
+1 & \text { for ordinary particles, } \\
-1 & \text { for superpartners. }
\end{array}\right.
\end{aligned}
$$

But why should we limit ourselves to the discrete $R$-parity symmetry, rather than considering its full continuous parent $R$-invariance? This continuous $U(1) \quad R$-invariance, from which $R$ parity has emerged, is indeed a symmetry of all four necessary basic building blocks of the Supersymmetric Standard Model [ 5]:

1) the Lagrangian density for $S U(3) \times S U(2) \times$ $U(1)$ gauge superfields;

2) the $S U(3) \times S U(2) \times U(1)$ gauge interactions of the quark and lepton superfields;

$3)$ the $S U(2) \times U(1)$ gauge interactions of the two chiral doublet Higgs superfields $H_{1}$ and $H_{2}$ responsible for the electroweak breaking;

4) and the trilinear "superYukawa" interactions (12) responsible for quark and lepton masses.

Indeed the bilinear products of left-handed (anti)lepton and (anti)quark superfields $\bar{E} L$, $\bar{D} Q$ and $\bar{U} Q$ that we have to consider to generate quark and lepton mass terms in a supersymmetric extension of the standard model transform under $R$ as follows, according to eq. (13):

$\bar{E} L(x, \theta) \stackrel{R}{\longrightarrow} e^{2 i \alpha} \bar{E} L\left(x, \theta e^{-i \alpha}\right), \ldots$

When these terms are combined with $H_{1}$ (for $\bar{E} L$ and $\bar{D} Q$ ) and $H_{2}$ (for $\bar{U} Q$ ) to give the trilinear superpotential (12), this one transforms under the continuous $R$-symmetry (13) with " $R$ - 
Table 3

$R$-parities in the Supersymmetric Standard Model.

\begin{tabular}{|c|c|}
\hline Bosons & Fermions \\
\hline \hline $\begin{array}{c}\text { gauge and Higgs bosons } \\
\text { graviton }\end{array}$ & $(R= \pm 1)$ \\
$(R=0)$ & $R$-parity - \\
$R$-parity + & gravitino \\
sleptons and squarks & $(R=0)$ \\
$(R= \pm 1)$ & $R$-parity + \\
$R$-parity - & \\
\hline
\end{tabular}

weight" $n_{\mathcal{W}}=\sum_{i} n_{i}=2$, i.e. according to

$\mathcal{W}(x, \theta) \stackrel{R}{\longrightarrow} e^{2 i \alpha} \mathcal{W}\left(x, \theta e^{-i \alpha}\right)$.

Its auxiliary " $F$-component" (obtained from the coefficient of the bilinear $\theta \theta$ term in the expansion of $\mathcal{W}$ ), is therefore $R$-invariant, generating $R$-invariant interaction terms in the Lagrangian density ${ }^{11}$.

However, an unbroken continuous $R$ invariance, which acts chirally on the Majorana octet of gluinos,

$\tilde{g} \stackrel{R}{\longrightarrow} e^{\gamma_{5} \alpha} \tilde{g}$.

would constrain gluinos to remain massless, even after a breaking of the supersymmetry. We would then expect the existence of relatively light " $R$ hadrons" [8, 9] made of quarks, antiquarks and gluinos, which have not been observed. We know today that gluinos, if they do exist, should be

\footnotetext{
${ }^{11}$ Note, however, that a direct Higgs superfield mass term $\mu H_{1} H_{2}$ in the superpotential, which has $R$-weight $n=0$, does not lead to interactions which are invariant under the continous $R$ symmetry; but it gets in general reallowed, as for example in the MSSM, as soon as the continuous $R$ symmetry gets reduced to its discrete version of $R$-parity.
}

rather heavy, requiring a significant breaking of the continuous $R$-invariance, in addition to the necessary breaking of the supersymmetry. Once the continuous $R$-invariance is abandoned, and supersymmetry is spontaneously broken, radiative corrections do indeed allow for the generation of gluino masses [21], a point to which we shall return later (cf. section 6 ).

Furthermore, the necessity of generating a mass for the Majorana spin- $\frac{3}{2}$ gravitino, once local supersymmetry is spontaneously broken, also forces us to abandon the continuous $R$-invariance, in favor of the discrete $R$-parity symmetry, thereby also allowing for gluino and other gaugino masses, at the same time as the gravitino mass $m_{3 / 2}$, as already noted in 1977 [ [6]. A third reason for abandoning the continuous $R$-symmetry could now be the non-observation at LEP of a charged wino - also called chargino - lighter than the $W^{ \pm}$, that would exist in the case of a continuous $U(1) R$-invariance [ [A, 5], as shown by the mass matrix $\mathcal{M}$ of eq. (8) (the just-discovered $\tau^{-}$particle could tentatively be considered, in 1976, as a possible light wino/chargino candidate, before getting clearly identified as a sequential heavy lepton.)

Once we drop the continuous $R$-invariance in favor of its discrete $R$-parity version, we may ask how general is this notion of $R$-parity, and, correlatively, are we forced to have this $R$-parity conserved? As a matter of fact, there is from the beginning a close connection between $R$-parity and baryon and lepton number conservation laws, which has its origin in our desire to get supersymmetric theories in which $B$ and $L$ could be conserved, and, at the same time, to avoid unwanted exchanges of spin-0 squarks and sleptons. Actually the superpotential of the theories discussed in Ref. [ [] was constrained from the beginning, for that purpose, to be an even function of quark and lepton superfields, more specifically involving, for left-handed electroweak doublets and right-handed singlets, the bilinear combinations $\bar{E} L, \bar{D} Q$ and $\bar{U} Q$. Odd superpotential terms, which would have violated the "matter-parity" symmetry $(-1)^{(3 B+L)}$, were excluded, to be able to recover $B$ and $L$ conser- 
vation laws, and avoid direct Yukawa exchanges of spin-0 squarks and sleptons between ordinary quarks and leptons. Tolerating unnecessary superpotential terms which are odd functions of quark and lepton superfields (i.e. $R_{p}$-violating terms), does create, in general, immediate problems with baryon and lepton number conservation laws (most notably, a much too fast proton instability, if both $B$ and $L$ violations are simultaneously allowed).

This intimate connection between $R$-parity and $B$ and $L$ conservation laws can be made quite obvious by noting that for usual particles $(-1)^{2 S}$ coincides with $(-1)^{3 B+L}$, so that the original $R$-parity (14) may be reexpressed in terms of the spin $S$ and the "matter-parity" $(-1)^{3 B+L}$, as follows [8]:

$R$-parity $=(-1)^{2 S}(-1)^{3 B+L}$.

This may also be written as $(-1)^{2 S}(-1)^{3(B-L)}$, showing that this discrete symmetry may still be conserved even if baryon and lepton numbers are separately violated, as long as their difference $(B-L)$ remains conserved, at least modulo 2 .

The $R$-parity symmetry operator may also be viewed as a non-trivial geometrical discrete symmetry associated with a reflection of the anticommuting fermionic Grassmann coordinate, $\theta \rightarrow$ $-\theta$, in superspace [22]. This $R$-parity operator plays an essential rôle in the discussion of the experimental signatures of the new particles (even if it should turn out not to be exactly conserved). A conserved $R$-parity guarantees that the new spin-0 squarks and sleptons cannot be directly exchanged between ordinary quarks and leptons, as well as the absolute stability of the "lightest supersymmetric particle" (or LSP), a good candidate for non-baryonic Dark Matter in the Universe.

\section{ABOUT SUPERSYMMETRY BREA- KING, IN THE EARLY TIMES}

Let us come back to the question of supersymmetry breaking, which still has not received a definitive answer yet. The inclusion, in the Lagrangian density, of universal soft dimension-
2 supersymmetry breaking terms for all squarks and sleptons,

$\mathcal{L}_{\text {soft }}=-\sum_{\tilde{q}, \tilde{l}} m_{0}^{2}\left(\tilde{q}^{\dagger} \tilde{q}+\tilde{l}^{\dagger} \tilde{l}\right)$,

was already considered in 1976. Such terms breaking explicitly the supersymmetry, as soon as they are allowed or tolerated, can immediately make superpartners heavy with no difficulty, in supersymmetric extensions of the standard model with an $S U(3) \times S U(2) \times U(1)$ gauge group (no extra $U(1)$ ), and quarks and charged leptons massive through their couplings to $H_{1}$ and $H_{2}$ [5]. These models may involve an additional singlet superfield $N$ coupled to $H_{1}$ and $H_{2}$ as in [ [A], with a trilinear coupling $\lambda H_{1} H_{2} N+\ldots$ in place of the initial $\mu H_{1} H_{2}$ (cf. "NMSSM"). One may also disregard this extra superfield, then restoring the $\mu H_{1} H_{2}$ mass term, which leads to the minimal version of the Supersymmetric Standard Model ${ }^{2}$.

We were however more ambitious, since it was also understood that such soft-breaking terms of dimension 2 should better originate from a spontaneous supersymmetry breaking mechanism, especially if supersymmetry is to be realized locally. This required additional work. As a matter of fact, in view of progressing towards a true spontaneous breaking mechanism, the soft breaking terms of eq. (19) were first generated spontaneously with the help of the " $D$-term" associated with an "extra $U(1)$ " gauge symmetry, acting axially on lepton and quark fields, thereby providing a common positive mass ${ }^{2}$ contribution $m_{0}^{2}$ (that was initially called $\mu^{2}$ ) for all ("lefthanded" as well as "right-handed") slepton and squark fields. One can then disregard this potentially unpleasant extra $U(1)$ by sending it to an "invisible sector", ultimately taking the limit in which its gauge coupling $g^{\prime \prime}$ vanishes, as done in the first paper of ref. [5]. The spin- $\frac{1}{2}$ goldstino (here the gaugino of the extra $U(1))$ gets "invisible" then decouples. The supersymmetry, first spontaneously broken but "at a very high scale"

\footnotetext{
${ }^{12}$ In both cases, the photon and gluons remain massless as they should, and there is no massless or light spin- 0 boson since no spontaneously broken extra $U(1)$ is present, once the singlet $N$ and/or the various soft-breaking terms are included.
} 
(here $\left.\sqrt{d}=\Lambda_{s s} \approx \sqrt{m_{0}^{2} / g^{\prime \prime}} \gg m_{W}\right)$, remains broken - now explicitly - in the limit; but only softly, through the slepton and squark (universal, in the simplest case) dimension-2 mass terms (19). The approach illustrated also very well the special rôle played by the "susy-breaking scale parameter", which could well be very large while the mass splittings between bosons and fermions still remain much smaller, typically $\lesssim$ electroweak scale.

Considering such an extra $U(1)$ is indeed natural in the framework of supersymmetric theories, in which two doublet Higgs superfields are separately responsible for the masses of charged leptons and down quarks $\left(H_{1}\right)$, and of up quarks $\left(H_{2}\right)$, as we have seen. One may therefore take advantage of this special feature of supersymmetric theories, also imposed by the necessity of avoiding massless charged fermions, to perform independent phase transformations on the two Higgs doublets. More precisely one can perform $U(1)_{Y} \times$ extra- $U(1)$ gauge transformations, acting independently on the two Higgs doublets $H_{1}$ and $H_{2}$. This corresponds to the invariance of the trilinear superpotential (12) responsible for quark and lepton masses under an extra $U(1)$ symmetry defined, in the simplest case, as follows:

$$
\begin{gathered}
\text { Action of extra } U(1): \\
\left\{\begin{array}{l}
V(x, \theta, \bar{\theta}) \longrightarrow V(x, \theta, \bar{\theta}), \\
H_{1,2}(x, \theta) \longrightarrow e^{-i \alpha} H_{1,2}(x, \theta), \\
S(x, \theta) \longrightarrow e^{i \frac{\alpha}{2}} S(x, \theta),
\end{array}\right. \\
\text { for }\left\{\begin{array}{c}
S U(3) \times S U(2) \times U(1) \text { gauge superfields, } \\
\text { left-h. Higgs superfields } H_{1} \text { and } H_{2}, \\
\text { left-h. (anti)quark and lepton superfields } \\
S=\{Q, \bar{U}, \bar{D}, L, \bar{E}\} \cdot
\end{array}\right.
\end{gathered}
$$

This extra $U(1)$ is associated, in the above simple case, with a purely axial new neutral current for all quarks and charged leptons 13. The whole

\footnotetext{
13 When we also introduce the extra neutral singlet chiral superfield $N$ coupled to $H_{1}$ and $H_{2}$, it gets transformed
}

construction is designed so as to make the set of equations $\langle D\rangle^{\prime} \mathrm{s}=\langle F\rangle^{\prime} \mathrm{s}=\langle G\rangle^{\prime} \mathrm{s}=0$ to have no solution at all, in order to obtain a true spontaneous breaking of the supersymmetry with a physically coupled goldstino (later to be "eaten" by the gravitino), as well a spontaneous breaking of the extra $U(1)$ and of the electroweak symmetry, with the $S U(3) \times U(1)$ of QCD $\times$ QED remaining unbroken: quite a non-trivial result given all the constraints to be satisfied simultaneously. The soft breaking terms $m_{0}^{2}$ of eq. (19) are then generated by the v.e.v. of the $D$-component associated with the extra $U(1)$, which reads in the simplest case:

$m_{0}^{2}=\frac{1}{2} g^{\prime \prime}<-D^{\prime \prime}>$,

so that one gets the mass relations:

$$
\left\{\begin{array}{l}
m_{\tilde{q}}^{2}=m_{q}^{2}+m_{0}^{2}, \\
m_{\tilde{l}}^{2}=m_{l}^{2}+m_{0}^{2} .
\end{array}\right.
$$

Additional contributions to $m_{0}^{2}$, which depend linearly on the weak isospin and hypercharge quantum numbers $T_{3}$ and $Y$, may also be generated from non-vanishing contributions of the $<D>$ 's associated with $S U(2) \times U(1)$, but they remain family-independent (which is useful to avoid potential difficulties with flavor-changing neutral current effects). In the presence of such terms, the previous formula (22) becomes:

$$
\begin{aligned}
m_{\tilde{q}, \tilde{l}}^{2}= & m_{q, l}^{2}+m_{0}^{2} \\
& \mp\left(g T_{3}<D_{3}>+\frac{g^{\prime}}{2} Y<D^{\prime}>\right),
\end{aligned}
$$

in which the sign $\mp$ corresponds to the two different (left and right, respectively) handedness of the sfermion fields considered.

This illustrates clearly the reason for having introduced such an extra $U(1)$, in order to get spontaneous supersymmetry breaking with

under the extra $U(1)$ as follows:

$$
N(x, \theta) \longrightarrow e^{2 i \alpha} N(x, \theta)
$$

so that its trilinear superpotential coupling $\lambda H_{1} H_{2} N$ be invariant under the extra $U(1)$. (A superpotential term linear in $N$, if present as in [ [- $]$, would however break explicitly the extra $U(1)$ symmetry, as would also do $N^{2}$ or $N^{3}$ terms.) 
heavy squarks and sleptons. Indeed in a spontaneously broken globally supersymmetric theory with $S U(3) \times S U(2) \times U(1)$ (or a fortiori $S U(5)$ ) as the gauge group, possible supersymmetrybreaking contributions to the mass ${ }^{2}$ matrices of squarks and sleptons originating from the weak hypercharge and weak isospin " $D$-terms" in the scalar potential always depend linearly on the $Y$ and $T_{3}$ gauge quantum numbers of (lefthanded) matter superfields, and therefore cannot all be positive; and $F$-term contributions mixing squarks or sleptons together cannot come to the rescue. As a result one at least among the four $\tilde{u}$ and $\tilde{d}$ squarks would then have a negative mass ${ }^{2}$ (unless they all remain light), which is evidently unacceptable. This is well illustrated by the mass sum rule established in [23], which reads, in the absence of such an extra $U(1)$ :

$\sum m^{2}$ (squarks) $=2 \sum m^{2}$ (quarks).

The average mass ${ }^{2}$ is the same for squarks and quarks, for example (up to radiative corrections), in a spontaneously broken globally supersymmetric theory, in the absence of an extra $U(1)$. The use of a "non-traceless" extra $U(1)$, as we had introduced a few years before precisely for that reason, allowed for a non-vanishing supertrace:

Supertrace $\mathcal{M}_{\text {(squarks and quarks) }}^{2}$

$$
\begin{aligned}
& =\sum m^{2} \text { (squarks) }-2 \sum m^{2} \text { (quarks) } \\
& \approx g^{\prime \prime}<D_{\text {extra- } U(1)}>\operatorname{Tr}_{\text {quark sf. }} \text { extra- } U(1)>\mathbf{0} ;
\end{aligned}
$$

and for heavy squarks and sleptons, already at the classical level, in spontaneously broken global supersymmetry.

The problem of having all squarks and sleptons heavy, in a spontaneously broken supersymmetric theory, appears solved for the moment, at the price of the extension of the gauge group to include an extra $U(1)$ factor. Otherwise one would have to rely, as it was proposed later, on rather complicated effects of radiative corrections, or to go to supergravity theories. Of course, in all cases one may always decide to return to explicit supersymmetry breaking, which immediately leads us to physically acceptable $S U(3) \times S U(2) \times U(1)$ theories, without or with additional neutral singlet chiral superfields. This makes the difficult question of supersymmetry breaking immediately disappear, although one still has to wonder about the physical origin of this breaking.

The previous method of spontaneous supersymmetry breaking quickly faced several difficulties. In addition to the question of anomalies (connected with the non-traceless feature of the extra $U(1)$, but maybe anomalous $U(1)$ 's could be tolerated after all ...), it required new neutral current interactions. This was fine in 1977, but such interactions did not show up, as the $S U(2) \times U(1)$ neutral current structure of the Standard Model got brilliantly confirmed. (The question is in fact more complex than it seems, in particular since one may also consider situations for which the new gauge boson would be both very light and very weakly coupled, but we do not elaborate on this here.) This mechanism also left us with the question of generating large gluino masses, to which we shall return soon. Altogether, the gauging of an extra $U(1)$ no longer appears as an appropriate way to generate large superpartner masses - even if such an $U(1)$ may still have a rôle to play - and we can now close this parenthesis. One now uses again, in general, soft supersymmetry-breaking terms [24] generalizing those of eq. (19) - possibly "induced by supergravity", or other mechanisms for which the supersymmetry is in general spontaneously broken "at a high scale". These terms essentially serve as a parametrization of our ignorance about the true mechanism of supersymmetry breaking chosen by Nature to make superpartners heavy.

But let us return to gluino masses. As we said before continuous $R$-symmetry transformations act chirally on gluinos, so that an unbroken $R$-invariance would require them to remain massless, even after a spontaneous breaking of the supersymmetry! Thus the need, once it became experimentally clear that massless or even light gluinos could not be tolerated, to generate a gluino mass either from radiative corrections [21], or from supergravity (see already [6]), with, in both cases, the continuous $R$-invariance reduced to its discrete $R$-parity subgroup. 
In the framework of global supersymmetry it is not so easy to generate large gluino masses. Even if global supersymmetry is spontaneously broken, and if the continuous $R$-symmetry is not present, it is still in general rather difficult to obtain large masses for gluinos, since: i) no direct gluino mass term is present in the Lagrangian density; and ii) no such term may be generated spontaneously, at the tree approximation, since gluino couplings involve colored spin-0 fields, which cannot be translated. A gluino mass may then be generated by radiative corrections involving a new sector of quarks sensitive to the source of supersymmetry breaking [21], that would now be called "messenger quarks", but iii) this can only be through diagrams which "know" both about:

a) the spontaneous breaking of the global supersymmetry, through some appropriately-generated v.e.v.'s for auxiliary components, $\langle D\rangle,\langle F\rangle$ or $\langle G>$ 's; b) the existence of superpotential interactions which do not preserve the continuous $U(1) R$-symmetry. Such radiativelygenerated gluino masses, however, generally tend to be rather small, unless one introduces, in some often rather complicated "hidden sector", very large mass scales $\gg m_{W}$.

Fortunately gluino masses may also result directly from supergravity, as already observed in 1977 [ 6]. Gravitational interactions require, within local supersymmetry, that the spin2 graviton be associated with a spin- $3 / 2$ partner [ 16], the gravitino. Since the gravitino is the fermionic gauge particle of supersymmetry it must acquire a mass, $m_{3 / 2}(=\kappa d / \sqrt{6} \approx$ $\left.d / m_{\text {Planck }}\right)$, as soon as the local supersymmetry gets spontaneously broken. Since the gravitino is a self-conjugate Majorana fermion its mass breaks the continuous $R$-invariance which acts chirally on it, just as for the gluinos, forcing us to abandon the continuous $U(1) R$-invariance, in favor of its discrete $R$-parity subgroup. In particular, in the presence of a spin- $\frac{3}{2}$ gravitino mass term $m_{3 / 2}$, which corresponds to a change in $R \quad \Delta R= \pm 2$, the "left-handed sfermions" $\tilde{f}_{L}$, which carry $R=+1$, can mix with the "righthanded" ones $\tilde{f}_{R}$, carrying $R=-1$, through mixing terms having $\Delta R= \pm 2$, which may naturally (but not necessarily) be of order $m_{3 / 2} m_{f}$
- so that the lightest of the sfermions may well turn out to be one of the two stop quarks $\tilde{t}$. Supergravity theories also offer, in addition, a natural framework in which to include direct gaugino Majorana mass terms

$-\frac{i}{2} m_{3} \overline{\tilde{G}}_{a} \tilde{G}_{a}-\frac{i}{2} m_{2} \quad \overline{\tilde{W}}_{a} \tilde{W}_{a}-\frac{i}{2} m_{1} \overline{\tilde{B}} \tilde{B}$,

which also correspond to $\Delta R= \pm 2$, just as for the gravitino mass itself. The $S U(3) \times S U(2) \times$ $U(1)$ gaugino mass parameters $m_{3}, m_{2}$ and $m_{1}$ may naturally (but not necessarily) be of the same order as the gravitino mass $m_{3 / 2}$.

Once the continuous $R$-invariance is reduced to its discrete $R$-parity subgroup, a direct Higgs superfield mass term $\mu \mathrm{H}_{1} \mathrm{H}_{2}$, which was not allowed by the continuous $U(1) R$-symmetry, gets reallowed in the superpotential, as for example in the MSSM. The size of this supersymmetric $\mu$ parameter might conceivably have been a source of difficulty, in case this parameter, present even if there is no supersymmetry breaking, turned out to be large. But since the $\mu$ term breaks explicitly both the continuous $R$-invariance (13) and the (global) extra $U(1)$ symmetry (20) its size may be controlled by considering one or the other of these two symmetries. Even better, since $\mu$ got reallowed just as we abandoned the continuous $R$-invariance so as to allow for gluino and gravitino masses, the size of $\mu$ may naturally be of the same order as these gaugino (and gravitino) masses, since they all appear in violation of the continuous $R$-symmetry (13). Altogether there is here no specific hierarchy problem associated with the size of $\mu$. In general, irrespective of the supersymmetry breaking mechanism considered, still unknown (and generally parametrized using a variety of possible soft supersymmetry breaking terms), one normally expects the various superpartners not to be too heavy, otherwise the corresponding new mass scale introduced in the game would tend to contaminate the electroweak scale, thereby creating a hierarchy problem in the Supersymmetric Standard Model. Superpartner masses are then normally expected to be naturally of the order of $m_{W}$, or at most in the $\sim$ $\mathrm{TeV} / c^{2}$ range. 
The Supersymmetric Standard Model ("minimal" or not), with its $R$-parity symmetry (absolutely conserved, or not), provided the basis for the experimental searches for the new superpartners and Higgs bosons, starting with the first searches for gluinos and photinos, selectrons and smuons, at the end of the seventies. How the supersymmetry should actually be broken, if indeed it is a symmetry of Nature, is not known yet. Many good reasons to work on the Supersymmetric Standard Model and its various extensions have been discussed, dealing with Dark Matter, supergravity, gauge coupling unification, extended supersymmetry, new spacetime dimensions, superstrings, " $M$-theory", $\ldots$. Despite all the efforts made for more than twenty years to discover the new inos and sparticles, we are still waiting for experiments to disclose this missing half of the SuperWorld. Still supersymmetry may well be, beyond quantum physics and general relativity, the next fundamental symmetry to be discovered in the physics of fundamental particles and interactions, enlarging our vision of space and time to the new anticommuting dimensions of superspace.

\section{REFERENCES}

1. Yu. A. Gol'fand and E.P. Likhtman, ZhETF Pis. Red. 13 (1971) 452 [JETP Lett. 13 (1971) 323].

2. D.V. Volkov and V.P. Akulov, Phys. Lett. B 46 (1973) 109.

3. J. Wess and B. Zumino, Nucl. Phys. B 70 (1974) 39; Phys. Lett. B 49 (1974) 52; Nucl. Phys. B 78 (1974) 1.

4. P. Fayet, Nucl. Phys. B 90 (1975) 104.

5. P. Fayet, Phys. Lett. B 64 (1976) 159; B 69 (1977) 489.

6. P. Fayet, Phys. Lett. B 70 (1977) 461.

7. P. Fayet, in New Frontiers in High-Energy Physics, Proc. Orbis Scientiae, Coral Gables (Florida, USA), 1978, eds. A. Perlmutter and L.F. Scott (Plenum, N.Y., 1978) p. 413.

8. G.R. Farrar and P. Fayet, Phys. Lett. B 76 (1978) 575.

9. G.R. Farrar and P. Fayet, Phys. Lett. B 79 (1978) 442.
10. G.R. Farrar and P. Fayet, Phys. Lett. B 89 (1980) 191.

11. P. Ramond, Phys. Rev. D 3 (1971) 2415 (1971); A. Neveu and J. Schwarz, Nucl. Phys. B 31 (1971) 86.

12. J.-L. Gervais and B. Sakita, Nucl. Phys. B 34 (1971) 632.

13. J. Iliopoulos and B. Zumino, B 76 (1974) 310.

14. P. Fayet and J. Iliopoulos, Phys. Lett. B 51 (1974) 461.

15. P. Fayet, Phys. Lett. B 58 (1975) 67; L. O'Raifeartaigh, Nucl. Phys. B 96 (1975) 331.

16. S. Ferrara, D.Z. Freedman and P. van Nieuwenhuizen, Phys. Rev. D 13 (1976) 3214; S. Deser and B. Zumino, Phys. Lett. B 62 (1976) 335.

17. E. Cremmer et al., Phys. Lett. B 147 (1979) 105.

18. P. Fayet, Phys. Lett. B 86 (1979) 272; B 175 (1986) 471.

19. P. Fayet, Nucl. Phys. B 78 (1974) 14.

20. M. Gell-Mann, P. Ramond and R. Slansky, Rev. Mod. Phys. 50 (1978) 721.

21. P. Fayet, Phys. Lett. B 78 (1978) 417.

22. P. Fayet, in History of original ideas and basic discoveries in Particle Physics, eds. H. Newman and T. Ypsilantis, Proc. Erice Conf., NATO Series B 352 (Plenum, N.Y., 1996) p. 639.

23. P. Fayet, Phys. Lett. B 84 (1979) 416.

24. L. Girardello and M.T. Grisaru, Nucl. Phys. B 194 (1982) 65. 